\title{
A Formalism for the Investigation of Algebraically Special Metrics. II
}

\author{
A. Held \\ Institut für Theoretische Physik der Universität Bern, CH-3000 Bern, Switzerland
}

Received July 15,1974

\begin{abstract}
The equations of the formalism developed in part I are simplified by specialisation of the basic pair of null directions. The auxiliary vectors previously introduced are shown to have intrinsic geometric properties which are directly related to the complexity of the differential operators of the formalism. An Ansatz based on these properties is introduced and the calculation of the metric so defined is used to display the connection between the coordinate-free and coordinate-dependent solutions.
\end{abstract}

\section{Introduction}

In this first paper [1] of this series a new technique for the investigation of empty space algebraically special solutions of the Einstein equations was introduced. In this work we develop the method further. We show that certain quantities which appear naturally in the course of calculation have a deeper significance and contain information about the intrinsic geometric structure of the space. We also demonstrate the efficacy of the method, showing how elementary arguments lead to simplifying Ansätze and how easily calculations may be done.

The first part of the paper contains a review of the method and a compact statement of the equations governing this type of metric. We show that for a particular choice of tetrad these equations may be partially integrated in a coordinate free sense and the problem thereby reduced to a set of eight linear equations, all of which are extremely simple in form.

The problem of simplifying specializations suggested by the previously derived equations are considered. This leads us to investigate the auxilliary vectors $\tilde{\alpha}$ and $\tilde{\beta}$ which were introduced in Ref. [1]. These vectors contain the information inherent in the Newman-Penrose (N.P.) spin coefficients [2] $\alpha, \beta, \gamma$, and $\varepsilon$, and are an essential part of the differential operators of the formalism. They are not of good weight [3] transforming inhomogeneously under the gauge transformations (cf. below), but despite this they contain important information concerning the intrinsic structure of the space and the solubility of the resulting equations is highly dependent on their properties.

The next part of the paper is devoted to a discussion of some properties common to all algebraically special empty-space metrics with special reference to a family of two surface metrics inherent in such solutions. Finally we derive the metric determined by a special Ansatz. 


\section{Section 2. Review of the Technique}

We deal with an algebra of objects and differential operators all of which are of good spin and boost weight in the sense of Ref. [3]. That is, given a standard null tetrad $l_{a}, n_{a}, m_{a}, \bar{m}_{a}$ (the bar represents complex conjugation) such that

$$
l^{a} n_{a}=-m^{a} \bar{m}_{a}=1
$$

and all other scalar products vanish, an object is said to be of weight $(p, q)$ if, under the tetrad gauge transformation.

$$
\begin{aligned}
& l^{a} \rightarrow \lambda l^{a} ; \quad n^{a} \rightarrow \lambda^{-1} n^{a}, \\
& m^{a} \rightarrow e^{i \theta} m^{a}, \\
& \eta \rightarrow \lambda^{t} e^{i s \theta} \eta
\end{aligned}
$$

where

$$
\begin{aligned}
& t=\frac{p+q}{2} \quad \text { is the boost weight } \\
& s=\frac{p-q}{2} \quad \text { is the spin weight. }
\end{aligned}
$$

The fundamental objects of the formalism are those elements of the N.P. spin coefficient formalism [2] which possess good weight. Following the practise initiated in Ref. [3], some of these will be designated differently than is customary. Those spin coefficients differing from the notation of Ref. [2] are (the original notation appears in brackets)

$$
\varrho^{\prime}(-\mu) ; \kappa^{\prime}(-v) ; \sigma^{\prime}(-\lambda) ; \tau^{\prime}(-\pi) \text {. }
$$

The differential operators are essentially those of Ref. [3], modified to take advantage of the simplified properties of algebraically special empty space metrics. To define the new operators we first introduce the auxilliary vectors

$$
\tilde{\alpha}_{a}=n^{b} \nabla_{a} l_{b}-\left[\tau \bar{\tau}\left(\frac{1}{\varrho}+\frac{1}{\varrho}\right)+\frac{1}{2}\left(\frac{\psi_{2}}{\varrho}+\frac{\bar{\psi}_{2}}{\varrho}\right)\right] l_{a}+\frac{\varrho}{\varrho} \bar{\tau} m_{a}+\frac{\bar{\varrho}}{\varrho} \tau \bar{m}_{a}
$$

and

$$
\tilde{\beta}_{a}=\bar{m}^{b} \nabla_{a} m_{b}+\left[\Omega^{\circ} \tau \bar{\tau}+\frac{1}{2}\left(\frac{\psi_{2}}{\varrho}-\frac{\bar{\psi}_{2}}{\varrho}\right)\right] l_{a}-\frac{\varrho}{\varrho} \bar{\tau} m_{a}+\frac{\bar{\varrho}}{\varrho} \tau \bar{m}_{a},
$$

where

$$
\Omega^{\circ}=\frac{1}{\varrho}-\frac{1}{\varrho} .
$$

The derivative operators $P, \tilde{\mathrm{D}^{\prime}}, \tilde{\delta}, \tilde{\sigma^{\prime}}$ are then defined by

$$
\nabla_{a}-t \tilde{\alpha}_{a}+s \hat{\beta}_{a}=l_{a}\left(\tilde{\mathrm{P}}^{\prime}+\bar{\tau} \tilde{\delta}+\tau \tilde{\delta}\right)+n_{a} \mathrm{P}-\varrho m_{a} \tilde{\partial}^{\prime}-\bar{\varrho} \bar{m}_{a} \tilde{\delta} .
$$

The vectors $\tilde{\alpha}_{a}$ and $\tilde{\beta}_{a}$ are not of good weight, transforming inhomogeneously under (2.2). However the combination of terms appearing on the L.H.S. of (2.6) 
does have weight $(0,0)$ so that the weights of $\mathrm{P}, \tilde{\mathrm{P}^{\prime}}, \tilde{\delta}$, and $\tilde{\delta}^{\prime}$ are $(1,1),(-1,-1)$, $(0,-2)$, and $(-2,0)$ respectively.

These operators have the properties that

$$
\left[\mathrm{P}, \tilde{\mathrm{P}}^{\prime}\right] \eta^{\circ}=[\mathrm{P}, \tilde{\delta}] \eta^{\circ}=\left[\mathrm{P}, \tilde{\delta}^{\prime}\right] \eta^{\circ}=0,
$$

if

$$
\mathrm{P} \eta^{\circ}=0 \text {. }
$$

(A degree sign ${ }^{\circ}$ will be used to mark any quantity annihilated by P.) In addition

$$
\tilde{\mathrm{P}}^{\prime}+\overline{\mathrm{P}}^{\prime}, \quad \overline{\tilde{O}}=\tilde{\delta}^{\prime} .
$$

For a more detailed treatment of the operators, including symmetry properties and the technique of integration without coordinates the reader is referred to Refs. $[1,3]$.

\section{Section 3. The Equations for a Particular Choice of Null Directions}

In Ref. [1] the N.P. equations [2] were rewritten for the algebraically special case using the operators $\mathrm{P}, \tilde{\mathrm{P}}^{\prime}, \tilde{\mathrm{O}}$, and $\tilde{\mathrm{O}}^{\prime}$. We display these equations again, but for a choice of tetrad which simplifies their form considerably. As usual the vector $l^{a}$ is chosen parallel to the degenerate principal null direction of the Weyl tensor. The vector $n^{a}$ is chosen so that $\tau=0$. This is always possible in the algebraically special case since under the null rotation ( $A$ is an arbitrary complex function)

$$
\begin{aligned}
& l^{a} \rightarrow l^{a}, m^{a} \rightarrow m^{a}+A l^{a} \\
& n^{a} \rightarrow n^{a}+A \bar{m}^{a}+\bar{A} m^{a}+A \bar{A} l^{a},
\end{aligned}
$$

$\tau$ transforms as

$$
\tau \rightarrow \tau+A \varrho
$$

$\varrho \neq 0$ is assumed throughout. This choice fixes the null directions uniquely.

From the field equations [1] it follows that $\tau^{\prime}=\sigma^{\prime}=0$. Taking this into account, the equations of Ref. [1] reduce to:

Field Equations

$$
\begin{aligned}
\mathrm{P} \varrho & =\varrho^{2}, \\
\mathrm{P} \kappa^{\prime} & =-\psi_{3}, \\
\mathrm{P} \varrho^{\prime} & =\varrho^{\prime} \bar{\varrho}-\psi_{2}, \\
\tilde{\delta} \varrho & =0, \\
\varrho \tilde{\delta}^{\prime} \varrho^{\prime} & =(\bar{\varrho}-\varrho) \kappa^{\prime}-\psi_{3}, \\
\tilde{\mathrm{P}}^{\prime} \varrho^{\prime}-\bar{\varrho} \tilde{\delta} \kappa^{\prime} & =-\frac{1}{2}\left[\frac{\psi_{2}}{\varrho}+\frac{\bar{\psi}_{2}}{\bar{\varrho}}\right] \varrho^{\prime}+\varrho^{2}, \\
\tilde{\tilde{O}^{\prime}} \kappa^{\prime} & =-\psi_{4} / \varrho, \\
\tilde{\mathrm{P}^{\prime}} \varrho & =\frac{1}{2}\left[\frac{\bar{\psi}_{2}}{\bar{\varrho}}-\frac{\psi_{2}}{\varrho}\right] \varrho+\varrho \bar{\varrho}^{\prime} .
\end{aligned}
$$


Bianchi Identities

$$
\begin{aligned}
& \mathrm{P} \psi_{2}=3 \varrho \psi_{2}, \\
& \mathrm{P} \psi_{3}-\varrho \tilde{\sigma}^{\prime} \psi_{2}=2 \varrho \psi_{3}, \\
& \mathrm{p} \psi_{4}-\varrho \tilde{\sigma^{\prime}} \psi_{3}=\varrho \psi_{4} \text {, } \\
& \tilde{\delta} \psi_{2}=0 \text {, } \\
& \tilde{\mathbf{P}}^{\prime} \psi_{2}-\bar{\varrho} \tilde{\partial} \psi_{3}=3 \varrho^{\prime} \psi_{2}, \\
& \tilde{\mathbf{p}}^{\prime} \psi_{3}-\bar{\varrho} \tilde{\delta} \psi_{4}=-3 \kappa^{\prime} \psi_{2}+\left[4 \varrho^{\prime}-\frac{\psi_{2}}{\varrho}\right] \psi_{3} .
\end{aligned}
$$

Commutators

$$
\begin{aligned}
{\left[\mathrm{P}, \tilde{\mathrm{P}}^{\prime}\right]=} & -\frac{1}{2}\left[\frac{\psi_{2}}{\varrho}+\frac{\bar{\psi}_{2}}{\bar{\varrho}}\right] \mathrm{P}, \\
{[\mathrm{P}, \tilde{\delta}]=} & 0 \\
{\left[\tilde{\delta}, \tilde{\delta}^{\prime}\right]=} & \frac{1}{\varrho \varrho} \bar{\varrho}\left(\bar{\varrho}^{\prime}-\varrho^{\prime}\right) \mathrm{P}+\Omega^{\circ} \tilde{\mathrm{P}}^{\prime}+p\left[\frac{\varrho^{\prime}}{\bar{\varrho}}+\frac{\psi_{2}}{2 \varrho}\left(\frac{1}{\varrho}+\frac{1}{\bar{\varrho}}\right)\right] \\
& +q\left[\frac{\bar{\varrho}^{\prime}}{\varrho}+\frac{\bar{\psi}_{2}}{2 \varrho}\left(\frac{1}{\varrho}+\frac{1}{\varrho}\right)\right], \\
{\left[\tilde{\mathrm{P}}^{\prime}, \tilde{\mathrm{O}}^{\prime}\right]=} & -\frac{\kappa^{\prime}}{\varrho} \mathrm{P}+p\left[\kappa^{\prime}+\frac{\psi_{3}}{\varrho}-\frac{1}{2} \tilde{\delta}^{\prime}\left(\frac{\psi_{2}}{\varrho}\right)\right]
\end{aligned}
$$

The remaining two commutators are obtained by complex conjugation of (3.7b) and (3.7d).

Using the integration techniques of Ref. [1] it is a simple matter to integrate these equations. The results are

$$
\begin{aligned}
& \varrho^{\prime}=\varrho \varrho^{\prime \circ}-\frac{1}{2}\left(\varrho^{2}+\varrho \varrho \bar{\varrho}\right) \psi_{2}^{\circ}, \\
& \kappa^{\prime}=\kappa^{\circ}-\varrho \psi_{3}^{\circ}-\frac{1}{2} \varrho^{2} \tilde{\delta}^{\prime} \psi_{2}^{\circ}-\frac{1}{2} \varrho^{3} \psi_{2}^{\circ}{\tilde{\sigma^{\prime}}}^{\circ}, \\
& \psi_{2}=\varrho^{3} \psi_{2}^{\circ} \text {, } \\
& \psi_{3}=\varrho^{2} \psi_{3}{ }^{\circ}+\varrho^{3} \tilde{\tilde{O}^{\prime}} \psi_{2}{ }^{\circ}+\frac{3}{2} \varrho^{4} \psi_{2}{ }^{\circ} \tilde{\delta}^{\prime} \Omega^{\circ}, \\
& \psi_{4}=\varrho \psi_{4}{ }^{\circ}+\varrho^{2} \tilde{\delta}^{\prime} \psi_{3}{ }^{\circ}+\varrho^{3}\left[\psi_{3}{ }^{\circ} \tilde{\delta^{\prime}} \Omega^{\circ}+\frac{1}{2} \tilde{\delta}^{\prime} \tilde{\delta}^{\prime} \psi_{2}{ }^{\circ}\right] \\
& +\varrho^{4}\left[\frac{3}{2} \tilde{\sigma^{\prime}} \psi_{2}{ }^{\circ} \tilde{\sigma}^{\prime} \Omega^{\circ}+\frac{1}{2} \psi_{2}{ }^{\circ} \tilde{O}^{\prime} \tilde{\partial}^{\prime} \Omega^{\circ}\right]+\frac{3}{2} \varrho^{5} \psi_{2}{ }^{\circ}\left(\tilde{O}^{\prime} \Omega^{\circ}\right)^{2},
\end{aligned}
$$

where $\varrho, \varrho^{\circ}, \kappa^{\circ}, \psi_{2}^{\circ}, \psi_{3}^{\circ}$, and $\psi_{4}^{\circ}$ are as yet undetermined functions.

The preceding expressions for the $\psi$ 's display Sachs' peeling theorem for algebraically special spacetimes. 
These functions are subject to the relations:

$$
\begin{aligned}
& {\tilde{\sigma^{\prime}}}^{\prime \circ} Q^{\circ}=-\left[\psi_{3}{ }^{\circ}+\Omega^{\circ} \kappa^{\prime \circ}\right] \text {, } \\
& \tilde{\mathrm{P}}^{\prime} Q^{\prime \circ}=\tilde{\partial} \kappa^{\prime \circ} \text {, } \\
& \tilde{\delta}^{\prime} \kappa^{\prime \circ}=-\psi_{4}^{\circ} \text {, } \\
& \tilde{\mathrm{o}} \psi_{4}{ }^{\circ}=\tilde{\mathrm{P}}^{\prime} \psi_{3}{ }^{\circ} \text {, } \\
& \tilde{\delta} \psi_{3}{ }^{\circ}=\tilde{\mathbf{P}}^{\prime} \psi_{2}{ }^{\circ} \text {, } \\
& \tilde{\partial} \psi_{2}{ }^{\circ}=0 \text {, } \\
& \tilde{ð \widetilde{\partial}^{\prime}} \Omega^{\circ}=2 \Omega^{\circ} \bar{\varrho}^{\prime \circ}+\psi_{2}{ }^{\circ}-\bar{\psi}_{2}^{\circ} \text {, } \\
& \tilde{\mathrm{P}}^{\prime} \Omega^{\circ}=\bar{\varrho}^{\prime \circ}-\varrho^{\prime \circ} \text {. }
\end{aligned}
$$

The derivative operators acting on $\varrho$ are

$$
\begin{aligned}
& \mathrm{P} \varrho=\varrho^{2} \quad \tilde{\mathrm{P}}^{\prime} \varrho=\varrho^{2} \bar{\varrho}^{\prime \circ}-\frac{1}{2} \varrho^{3} \psi_{2}{ }^{\circ}-\frac{1}{2} \varrho \varrho^{2} \bar{\psi}_{2}^{\circ} \\
& \tilde{\delta} \varrho=0 \quad \tilde{\delta^{\prime}} \varrho=Q^{2} \tilde{\delta}^{\prime} \Omega^{\circ}
\end{aligned}
$$

and the commutators reduce to

$$
\begin{aligned}
& {\left[\mathrm{P}, \tilde{\mathrm{P}}^{\prime}\right]=-\frac{1}{2}\left(\varrho^{2} \psi_{2}^{\circ}+\bar{\varrho}^{2} \bar{\psi}_{2}^{\circ}\right) \mathrm{P},} \\
& {[\mathrm{P}, \tilde{\sigma}]=0 \text {, }} \\
& {\left[\mathrm{P}, \tilde{\mathrm{O}^{\prime}}\right]=0 \text {, }} \\
& {\left[\tilde{\tilde{\sigma}}, \tilde{\sigma^{\prime}}\right]=\left[\frac{\bar{\varrho}^{\prime \circ}}{\bar{\varrho}}-\frac{\varrho^{\prime \circ}}{\varrho}+\frac{1}{2}\left(2+\varrho \Omega^{\circ}\right) \psi_{2}^{\circ}-\frac{1}{2}\left(2-\bar{\varrho} \Omega^{\circ}\right) \bar{\psi}_{2}^{\circ}\right] \mathrm{P}} \\
& +\Omega^{\circ} \tilde{\mathrm{P}}^{\prime}+p \varrho^{\prime \circ}-q \bar{\varrho}^{\prime \circ}, \\
& {\left[\tilde{\mathrm{p}}^{\prime}, \tilde{\delta}\right]=\left[-\frac{\bar{\kappa}^{\prime \circ}}{\bar{\varrho}}+\bar{\psi}_{3}^{\circ}+\frac{1}{2} \tilde{\varrho} \tilde{\tilde{\psi}} \bar{\psi}_{2}^{\circ}-\frac{1}{2} \bar{\varrho}^{2} \bar{\psi}_{2}^{\circ} \tilde{\delta} \Omega^{\circ}\right] \mathrm{P}+q \bar{\kappa}^{\prime \circ},} \\
& {\left[\tilde{\mathrm{E}}^{\prime},{\tilde{\delta^{\prime}}}^{\prime}\right]=\left[-\frac{\kappa^{\prime \circ}}{\varrho}+\psi_{3}{ }^{\circ}+\frac{1}{2} \varrho \tilde{\sigma}^{\prime} \psi_{2}^{\circ}+\frac{1}{2} \varrho^{2} \psi_{2}^{\circ}{\tilde{\sigma^{\prime}}}^{\circ}\right] \mathrm{P}+p \kappa^{\prime \circ} \text {. }}
\end{aligned}
$$

Equations (3.14)-(3.23) form the basis for the work to follow. We emphasize that the commutators are not identities, and in any solution they must be checked for consistency.

\section{Section 4. Specialisations}

A complete solution of the Eqs. (3.14)-(3.21) would represent the most general possible empty space algebraically special metric. To date this problem has proved intractable. Talbot [4] using the N.P. formalism has reduced the problem to a minimal set of differential equations (MDE) which while simpler than the original Einstein equations are still sufficiently frightening in appearance to quail even the stoutest of hearts. Various people have pushed the problem further through the use of simplifying Ansätze. The most useful of these were those which led Kerr to the metric which bears his name [5]. He assumed type D plus further simplifications to arrive at a unique metric. A simple Ansatz will in general produce a "class" of metrics. The assumption that the metric be of type D leads to a problem 
which was completely solved by Kinnersley [6]. A partially successful Ansatz was that of Robinson and Trautman [7], namely that the degenerate principle null vector should be hypersurface orthogonal (Equivalent to the assumption $\varrho=\bar{\varrho}$.). This enabled them to reduce the MDE of a class of solutions to a single one. Unfortunately it is of such a nature that ten years after its appearance only simple stationary solutions have been found.

Further successful Ansätze have been associated with the curvature of a two surface metric " $P$ " which invariably appears in the description of an algebraically special metric (cf. Section 6).

We will argue that to be reasonable, an Ansatz must be a gauge invariant statement. For example the Ansatz that a quantity $\eta$ shall be real (imaginary) can only have a non-trivial meaning if $\eta$ is type $(p, p)$, i.e., under the transformation (2.2)

$$
\eta \rightarrow \lambda^{p} \eta
$$

so that the condition that $\eta$ is real (imaginary) is maintained. Note that $\varrho$ is a $(1,1)$ quantity. Statements of the reality of objects not conforming to this rule or of the equality of quantities not of equal spin and boost weights are not genuine Ansätze but rather conditions imposed on the gauge. For example the Ansatz $\kappa^{\prime \circ}=\psi_{4}{ }^{\circ}$ is not really an Ansatz since this can always be satisfied by a particular choice of gauge. The Eqs. (3.14)-(3.21) represent a source of Ansätze. Aside from the obvious possibility of setting any of the fundamental variables equal to zero we could also choose to set terms involving the derivative operators equal to zero or even to equate two of them, always providing the wgts. agree. For example, a useful satz, which will be used in a subsequent paper, is $\overline{\mathrm{P}}^{\prime} \psi_{2}^{\circ}=0$.

There remains one further source of Ansatz, the auxiliary vectors $\tilde{\alpha}_{a}$ and $\tilde{\beta}_{a}$. It was mentioned previously that under gauge transformations these transform inhomogeneously. It is natural then to ask whether there exists a gauge in which they can be made to vanish. This is the problem with which the balance of this paper will be concerned. We will assume as our Ansatz that there exists a choice of gauge such that both $\tilde{\alpha}_{a}$ and $\tilde{\beta}_{a}$ vanish. This is a gauge invariant statement and so is a valid Ansatz according to the rule established above. To understand the ramifications of this assumption, the next section is devoted to an analysis of the transformation properties of $\tilde{\alpha}_{a}$ and $\tilde{\beta}_{a}$.

\section{Section 5. The Auxilliary Vectors}

We return now to the case of an arbitrary tetrad $(\tau \neq 0)$ and consider the vector $\tilde{\alpha}_{a}\left(\tilde{\beta}_{a}\right)$ as defined by (2.4). We first investigate its transformation properties under the three transformations associated with a null tetrad, one null direction of which remains fixed (in this case $l^{a}$ ). They are the transformations (2.2) and the null rotation (3.1). We find that the null rotation (3.1) leaves $\tilde{\alpha}_{a}\left(\tilde{\beta}_{a}\right)$ invariant so that modulo the transformations (2.2) the vector $\tilde{\alpha}_{a}\left(\tilde{\beta}_{a}\right)$ is uniquely determined.

Turning to the gauge transformations we see that under $(2.2 b) \tilde{\alpha}_{a}$ is invariant, but under (2.2a),

$$
\tilde{\alpha}_{a} \rightarrow \tilde{\alpha}_{a}+\lambda^{-1} \nabla_{a} \lambda \text {. }
$$


Hence the condition that $\tilde{\alpha}_{a}$ can be made to vanish by choice of gauge is equivalent to $\tilde{\alpha}_{a}$ being a gradient - a property invariant under (2.2a). If the vector $\tilde{\alpha}_{a}$ is "disposable" by choice of gauge, this property is totally independent of choice of tetrad (always provided that the direction of $l^{a}$ is that of the degenerate principle null direction) and so an intrinsic property of the space.

The necessary and sufficient conditions that $\tilde{\alpha}_{a}$ be "disposable" may be found through the direct and somewhat brutal calculation

$$
\tilde{\alpha}_{[a, b]}=0 \text {. }
$$

The conditions arrived at in this manner are

$$
\Sigma^{\circ}=\bar{\Sigma}^{\circ} \text { and } \Gamma^{\circ}=0,
$$

where

$$
\begin{aligned}
& \Sigma^{\circ}=\frac{\varrho^{\prime}}{\bar{\varrho}}+\frac{\psi_{2}}{2 \varrho}\left(\frac{1}{\varrho}+\frac{1}{\bar{\varrho}}\right)+\tilde{\delta}\left(\frac{\bar{\tau}}{\bar{\varrho}}\right), \\
& \Gamma^{\circ}=\kappa^{\prime}+\frac{\psi_{3}}{\varrho}+\tilde{P}^{\prime}\left(\frac{\bar{\tau}}{\bar{\varrho}}\right)-\frac{\bar{\tau} \psi_{2}}{\varrho \varrho}-\frac{\bar{\tau} \varrho^{\prime}}{\bar{\varrho}}-\frac{1}{2} \tilde{O}^{\prime}\left(\frac{\psi_{2}}{\varrho}\right)-\frac{\sigma^{\prime} \tau}{\varrho} .
\end{aligned}
$$

The expressions $\Sigma^{\circ}$ and $\Gamma^{\circ}$ were introduced in Ref. [1] where they appeared in the commutators

$$
\begin{aligned}
& {\left[\tilde{\delta}, \tilde{\delta}^{\prime}\right]=\frac{1}{\varrho \bar{\varrho}}\left(\bar{\varrho}^{\prime}-\varrho^{\prime}\right) \mathrm{P}+\Omega^{\circ} \tilde{\mathrm{P}}^{\prime}+p \Sigma^{\circ}-q \bar{\Sigma}^{\circ},} \\
& {\left[\tilde{\mathrm{P}^{\prime}}, \tilde{\delta}^{\prime}\right]=\left[\frac{\bar{\tau}}{\varrho \bar{\varrho}}\left(\varrho^{\prime}-\bar{\varrho}^{\prime}\right)-\frac{\kappa^{\prime}}{\varrho}\right] \mathrm{P}+p \Gamma^{\circ} .}
\end{aligned}
$$

From these considerations we would expect conditions (5.3) to be maintained under the null rotation (3.1). In fact, under (3.1)

$$
\Sigma^{\circ} \rightarrow \Sigma^{\circ} \text { and } \Gamma^{\circ} \rightarrow \Gamma^{\circ},
$$

so that both $\Sigma^{\circ}$ and $\Gamma^{\circ}$ are scalar fields defined to within a gauge transformation by the geometry of the metric. Their geometrical interpretation in terms of the tetrad chosen is not however invariant. For example the scalar field $\Sigma^{\circ}$ can be related to the asymptotic expansion properties of the congruence associated with $n^{a}$ for the choice of tetrad in Section 3, i.e. it is equivalent to $\varrho^{\prime}$. But this does not obtain for all choices of tetrad, and as yet no general geometric interpretation of either $\Gamma^{\circ}$ or $\Sigma^{\circ}$ has been found.

There is however a more elegant and useful way looking at the problem of the necessary and sufficient conditions (5.3). This alternative approach is summed up in the following assertion.

The following three statements are equivalent.
a) $\exists$ a gauge in which $\tilde{\alpha}_{a}=0$,
b) $\exists$ an object $\eta(p=q \neq 0)$ s.t.
$\mathrm{P} \eta=\tilde{\mathbf{P}^{\prime}} \eta=\tilde{\delta} \eta=\tilde{\delta^{\prime}} \eta=0$,
c) $\bar{\Sigma}^{\circ}-\Sigma^{\circ}=0, \Gamma^{\circ}=0$. 
Consider a) and b). For a ( $p, p)$ object, Eq. (2.6) reads

$$
\nabla_{a} \eta-p \tilde{\alpha}_{a} \eta=l_{a}\left[\tilde{\mathrm{P}}^{\prime}+\bar{\tau} \tilde{\partial}+\tau \tilde{\delta^{\prime}}\right] \eta+n_{a} \mathrm{P} \eta-\varrho m_{a} \tilde{\delta^{\prime}} \eta-\bar{\varrho} \bar{m}_{a} \tilde{\partial} \eta \text {. }
$$

Suppose that a) holds. Then in that gauge choose $\eta=$ constant and the L.H.S. of (5.9) vanishes. It follows that in that gauge (and hence in all gauges) b) holds. Similarly if $b$ ) holds for $\eta$ it also holds for

$$
\lambda=\eta+\bar{\eta} .
$$

But $\lambda$ is real and of $\operatorname{wgt}(p, p)$. Therefore it may be set equal to a constant by the transformation

$$
l^{a} \rightarrow \lambda^{-\frac{1}{p}} l^{a}, \quad n^{a} \rightarrow \lambda^{\frac{1}{p}} n^{a}
$$

In this gauge (2.6) reduces to

$$
-p \tilde{\alpha}_{a} \lambda=0
$$

and assertion a) holds.

Next we consider b) and c). If we assume b) and apply the commutation relationships of Ref. [1] to $\eta$ we find that they are automatically fulfilled with the exception of $\left[\tilde{\delta}, \tilde{\delta^{\prime}}\right] \eta,\left[\tilde{\mathrm{p}}^{\prime}, \tilde{\delta}^{\prime}\right] \eta$ and $\left[\tilde{\mathrm{p}}^{\prime}, \tilde{\tilde{\delta}^{\prime}}\right] \eta$. [Eqs. (5.6) and (5.7) of this article]. The requirement that they be fulfilled leads to $\mathrm{c}$ ).

In Ref. [8] it was shown that if any two of the operators $\mathbf{P}, \mathrm{P}^{\prime}, \circlearrowright^{\prime} ð^{\prime}$ of Ref. [3] form an involutive system for a given $p, q$, then there exists a non-trival object $\eta$ with $\operatorname{wgt}(p, q)$ which is annihilated by both operators. It is a simple matter to extend this result to the operators of this paper and to more than two operators. If we assume c), then for $p=q$ objects the operators $\mathrm{P}, \tilde{\mathrm{P}^{\prime}}, \tilde{\mathrm{\delta}}, \tilde{\mathrm{o}}^{\prime}$ form an involutive system and by the extension of the lemma of Ref. [8] there exists a $(p, p)$ object such that $b)$ is fulfilled.

This completes the proof of the assertion.

If we turn our attention to the vector $\tilde{\beta}_{a}$ we see that three equivalent conditions are

a) $\exists$ a gauge in which $\tilde{\beta}_{a}=0$,

b) $\exists$ an object $\eta(p=-q \neq 0)$ s.t.

$\mathrm{P} \eta=\tilde{\mathrm{P}^{\prime}} \eta=\tilde{\delta} \eta=\tilde{\delta^{\prime}} \eta=0$,

c) $\Sigma^{\circ}+\bar{\Sigma}^{\circ}=0, \Gamma^{\circ}=0$.

If b) holds, the gauge in which a) obtains is the one in which $\eta=\bar{\eta}$.

As stated in Section 4 , we are concerned with the case where both $\tilde{\alpha}_{a}$ and $\tilde{\beta}_{a}$ are disposable through choice of gauge. From the above we see that with our choice of tetrad, this is equivalent to

$$
\varrho^{\prime \circ}=\kappa^{\prime \circ}=0 \text {. }
$$

\section{Section 6. Calculation of the Metric}

We begin by considering (in an arbitrary tetrad) the vector

$$
m_{a}-\frac{\tau}{\varrho} l_{a}
$$


under the action of the null rotation (4.1). The behaviours of $m^{a}, l^{a}$ and $\tau$ are given by (3.1) and (3.2), and $\varrho$ is invariant. Combining these results we find that the vector (6.1) is invariant. Moreover it is hypersurface orthogonal. To see this, choose a tetrad such that $\tau=0$. Then (6.1) is simply $m_{a}$, and the conditions that it be proportional to a gradient are

$$
\sigma=\sigma^{\prime}=\tau-\bar{\tau}^{\prime}=0
$$

But with this choice of tetrad, these conditions are fulfilled (cf. Section 3). Therefore we may write

$$
-P\left(\bar{m}_{a}-\frac{\bar{\tau}}{\bar{Q}} l_{a}\right)=\zeta, a .
$$

For the choice of tetrad with which we will work $(\tau=0)$ this reduces to

$$
-P \bar{m}_{a}=\zeta,_{a}
$$

which defines $P$ modulo the redefinition of $\zeta$ by

$$
\zeta \rightarrow \zeta^{\prime}=\zeta^{\prime}(\zeta)
$$

Since

$$
g_{a b}=2 l_{(a} n_{b)}-2 m_{(a} \bar{m}_{b)}
$$

for this choice of tetrad, no matter what the co-ordinate system, the term

$$
\frac{2 d \zeta d \bar{\zeta}}{P \bar{P}}
$$

must necessarily appear in the co-variant form of the metric. Therefore $P$ determines a 2-parameter family of two dimensional positive define metrics although a family of 2-surfaces with these metrics as induced metrics appears naturally only in the Robinson-Trautman [7] case.

If we apply the commutators involving $\tilde{\delta}$ to the $(0,0)$ function $\zeta$ (all other commutators vanish identically) we derive equations [9] governing the " 2 metric" $P$.

$$
\begin{aligned}
& {[\mathrm{P}, \tilde{\delta}] \zeta=0 \Rightarrow P=\bar{\varrho} P^{\circ},} \\
& {\left[\tilde{\mathrm{P}}^{\prime}, \tilde{\delta}\right] \zeta=0 \Rightarrow \tilde{\mathrm{P}}^{\prime} P^{\circ}=0 \text {, }} \\
& {\left[\tilde{\tilde{O}^{\prime}}, \tilde{\delta}\right] \zeta=0 \Rightarrow \tilde{\delta}^{\prime} P^{\circ}=0 \text {. }}
\end{aligned}
$$

We choose $x^{3}=\zeta, x^{4}=\bar{\zeta}$. With this choice of co-ordinate system $l^{a}$ and $n^{a}$ are confined to a subspace of the tangent space spanned by $\frac{\partial}{\partial x^{1}}$, and $\frac{\partial}{\partial x^{2}}$. If the gauge be chosen so that $\tilde{\alpha}_{a}=\tilde{\beta}_{a}=0$, we have from (2.6) the equivalences

$$
\mathrm{P}=l^{a} \nabla_{a}, \quad \tilde{\mathrm{p}}^{\prime}=n^{a} \nabla_{a}, \quad \tilde{\mathrm{o}}=\frac{m^{a}}{\varrho} \nabla_{a}, \quad \tilde{\delta}^{\prime}=\frac{\bar{m}^{a}}{\varrho} \nabla_{a} .
$$


It follows then that

$$
\mathrm{P} \eta^{\circ}=\tilde{\mathrm{P}}^{\prime} \eta^{\circ}=0 \Rightarrow \eta^{\circ}=\eta^{\circ}(\zeta, \bar{\zeta}) \text {. }
$$

Turning now to the Eqs. (3.14)-(3.21), we see that the Ansatz $\varrho^{\prime \circ}=\kappa^{\prime \circ}=0$ implies

$$
\psi_{3}^{\circ}=\psi_{4}^{\circ}=0
$$

and the conditions

$$
\tilde{\mathrm{P}}^{\prime} \psi_{2}^{\circ}=\tilde{\mathrm{P}}^{\prime} \Omega^{\circ}=\tilde{\delta} \psi_{2}^{\circ}=0 \text {. }
$$

$\psi_{2}^{\circ}, \Omega^{\circ}$, and $P^{\circ}$ obey the requirements (6.9), so that they are functions of $\zeta$ and $\bar{\zeta}$ only. When acting on such a function, the operator $m^{a} \nabla_{a}\left(\bar{m}^{a} \nabla_{a}\right)$ reduces to $P \frac{\partial}{\partial \zeta}\left(\bar{P} \frac{\partial}{\partial \bar{\zeta}}\right)$. Using (6.7) and (6.11), $P^{\circ}$ and $\psi_{2}^{\circ}$ are then further restricted to

$$
P^{\circ}=P^{\circ}(\zeta), \quad \psi_{2}^{\circ}=\psi_{2}^{\circ}(\bar{\zeta}) .
$$

Finally, using the freedom of (6.4) we can set $P^{\circ}=1$. There remains now only the differential Eq. (3.20) to be satisfied. This takes the form

$$
\frac{\partial^{2} \Omega^{\circ}}{\partial \zeta \partial \bar{\zeta}}=\psi_{2}^{\circ}(\bar{\zeta})-\bar{\psi}_{2}^{\circ}(\zeta)
$$

Peeking ahead, we set

$$
\psi_{2}^{\circ}=\bar{G}^{\prime \prime}(\bar{\zeta}),
$$

then

$$
\Omega^{\circ}=\zeta \bar{G}^{\prime}(\bar{\zeta})-\bar{\zeta} G^{\prime}(\zeta)+F^{\prime}(\zeta)-\bar{F}^{\prime}(\bar{\zeta}),
$$

where $G$ and $F$ are arbitrary analytic functions of $\zeta$.

The solution is complete. We now have to choose the two co-ordinates $x^{1}$ and $x^{2}$ and determine the associated tetrad components. For $x^{2}$ we choose

$$
r=-\frac{1}{2}\left(\frac{1}{\varrho}+\frac{1}{\varrho}\right) .
$$

Using Eqs. (3.22) and (2.6), we arrive at

$$
\begin{aligned}
\delta_{a}^{2}= & n_{a}-\frac{1}{2}\left(\varrho \psi_{2}^{\circ}+\bar{\varrho} \bar{\psi}_{2}^{\circ}\right) l_{a}\left(-\frac{1}{2}\right) \varrho \frac{\partial}{\partial \bar{\zeta}} \Omega^{\circ} m_{a} \\
& +\frac{1}{2} \bar{\varrho} \frac{\partial}{\partial \zeta} \Omega^{\circ} \bar{m}_{a}
\end{aligned}
$$

an equation which gives $l^{2}, n^{2}, m^{2}, \bar{m}^{2}$ without further effort (cf. Ref. [1] for a more detailed treatment). 
We choose $x^{1}$ in the usual manner [10], i.e. we start with a coordinate surface $x$ such that $n^{1}=1$ and $l^{1}=0$. Then

$$
\begin{aligned}
& {[\mathrm{P}, \tilde{\delta}] \tilde{x}^{1}=0 \Rightarrow m^{1}=\varrho m^{1 \circ} \text {, }} \\
& {\left[\tilde{\mathrm{p}}^{\prime}, \tilde{\delta}\right] \tilde{x}^{1}=0 \Rightarrow \tilde{\mathbf{p}}^{\prime} m^{1 \circ}=0 \text {, }} \\
& {\left[\tilde{\delta}, \tilde{\delta}^{\prime}\right] \tilde{x}^{1}=\Omega^{\circ} \tilde{\mathbf{P}^{\prime}} \tilde{x} \Rightarrow \tilde{\delta} \bar{m}^{1 \circ}-{\tilde{\delta^{\prime}}}^{\prime 1 \circ}=\Omega^{\circ} \text {. }}
\end{aligned}
$$

We see that a general solution is

$$
m^{1 \circ}=M^{\circ}+\bar{F}-\zeta \bar{G},
$$

where $M^{\circ}$ is a solution of the homogeneous part of Eq. (6.18c). We remove this ambiguity in the coordinate system by a transformation of the form

$$
\tilde{x}^{1} \rightarrow x^{1}=\tilde{x}^{1}-\frac{1}{2} \int \frac{M^{\circ}}{P^{\circ}} d \zeta-\frac{1}{2} \int \frac{\bar{M}^{\circ}}{\bar{P}^{\circ}} d \bar{\zeta} .
$$

(The term $P^{\circ}$ is included in this last transformation even though in this particular case it is identically 1 . This renders the method of choosing a unique $x^{1}$ completely general. (The final form of the tetrad is then

$$
\begin{aligned}
& l^{a}=\{0, \quad 1, \quad 0, \quad 0\} \\
& n^{a}=\left\{1, \quad-\frac{1}{2}\left(\varrho \bar{G}^{\prime \prime}+\bar{\varrho} G^{\prime \prime}\right), \quad 0, \quad 0\right\} \\
& m^{a}=\left\{\bar{\varrho}(\bar{F}-\zeta \bar{G}), \quad-\frac{1}{2} \bar{\varrho} \frac{\partial}{\partial \zeta} \Omega^{\circ}, \quad \bar{\varrho}, \quad 0\right\},
\end{aligned}
$$

where $F(\zeta)$ and $G(\zeta)$ are arbitrary analytic functions.

\section{Conclusions}

In this paper we have continued our analysis of the method proposed in Ref. [1] for the investigation of empty space solutions of Einsteins equation with degenerate Weyl tensor. Attention was focused on the auxiliary vectors $\tilde{\alpha}_{a}$ and $\tilde{\beta}_{a}$ and some of their more important properties were identified. As a vehicle for further display of the technique a metric was derived based on the Ansatz that these vectors were "gauge disposable". This particular metric while not explicitly displayed is inherent in Ref. [11] where it would be characterized as being stationary and having a constant Gaussian curvature associated with its attendant two space metric $P^{\circ}$, along with a particular imposed co-ordinate condition.

\section{References}

1. Held, A.: Commun. math. Phys. 37, 311 (1974)

2. Newman, E. T., Penrose, R.: J. Math. Phys. 3, 566 (1962)

3. Geroch, R., Held, A., Penrose, R.: J. Math. Phys. 14, 874 (1973)

4. Talbot, C. J.: Commun. math. Phys. 13, 45 (1969) 
5. Kerr, R.P.: Phys. Rev. Lett. 11, 237 (1963)

6. Kinnersley, W.: J. Math. Phys. 10, 1195 (1969)

7. Robinson, I., Trautmann, A.: Proc. Roy. A 265, 462 (1962)

8. Held, A., Voorhees, B.: Intern J. of T.P. 10, 179 (1974)

9. The author is indebted to V. Blöchlinger for pointing this out

10. Newman, E., Tamborino, L., Unti, T.: J. Math. Phys. 4, 915 (1963)

11. Robinson, I., Robinson, J., Zund, J.: J. Math. and Mech. 18, 881 (1969)

Communicated by J. Ehlers 